ESAIM: Proceedings, Vol. 4, 1998, 269-283

Contrôle et Équations aux Dérivées Partielles

http://www.emath.fr/proc/Vol.4/

\title{
WELL-POSEDNESS ISSUES CONCERNING A MAGNETOSTRICTIVE ACTUATOR MODEL
}

\author{
RALPH C. SMITH \\ Department of Mathematics \\ Iowa State University \\ Ames, IA 50011 \\ E-mail: rsmith@iastate.edu
}

Key Words : Hysteresis Model, Magnetostrictive Materials.

AMS Subject Qualification : 73K50, 78A30, 93A30.

Article published by EDP Sciences and available at http://www.edpsciences.org/proc or http://dx.doi.org/10.1051/proc:1998033 


\begin{abstract}
Modeling issues concerning magnetostrictive actuators for structural systems are considered. Such actuators utilize the realignment of magnetic moments in response to applied magnetic fields to generate strains in the material. Strains and forces generated in this manner are significantly larger than those produced by many other smart materials but also exhibit significant nonlinearities and hysteresis. An energy-based model is employed here to characterize the inherent hysteresis and nonlinearities in a manner amenable to structural applications. Well-posedness issues are considered in the context of Euler-Bernoulli and Timoshenko beam models. This provides a framework which can be directly extended to more complex structures and is adequate for ensuing control applications.
\end{abstract}




\section{Introduction}

The development of new magnetostrictive materials has led to the advent of actuators which are advantageous in many structural and structural acoustic applications. These actuators utilize the property that strains in the material are generated in response to an applied magnetic field. In materials such as Terfenol-D, the strains and generated forces are sufficiently large to drive systems comprised of thick structures and heavy components. In such systems, magnetostrictive actuators are often advantageous over other smart material transducers such as piezoceramics and electrostrictives. The difficulty associated with magnetostrictive actuators lies in the hysteresis and nonlinearities inherent to the materials. This paper is concerned with providing a characterization of the material which can be utilized for ensuing control design.

To illustrate issues which must be addressed when modeling the material, we consider a typical magnetostrictive transducer as depicted in Figure 1. As detailed in [4], the primary components of the transducer include a magnetostrictive rod, a wound wire solenoid, and a cylindrical permanent magnet. The sensor/actuator capabilities of the magnetostrictive material are provided by magnetic moments which rotate in the presense of an applied magnetic field. As depicted in Figure 2a, the moments are primarily oriented perpendicular to the longitudinal rod axis in the absence of an applied field. Prestressing the rod with the spring washer serves to increase the number of moments perpendicular to the axis (see Figure 2b). When a field is applied in the direction of the rod axis, moments align in the sense depicted in Figure 2c and significant strains and forces are generated.

The driving field $H(t)$ is generated through a time-dependent current $\mathcal{I}(t)$ applied to the solenoid along with the field $H_{0}$ generated by the permanent magnet. To model the transducer for structural applications, it is necessary to characterize the relationship between the current $\mathcal{I}$ applied to the solenoid, the resulting field $H$, the associated magnetization $M$, and finally the generated strains $e$. As noted in Figure 3, the relationships are highly nonlinear and exhibit significant hysteresis.

A physics-based model characterizing the material is outlined in Section 2. In Section 3, the actuator model is illustrated in the context of Euler-Bernoulli and Timoshenko beam models. An abstract framework compatible with the unbounded actuator input is developed and well-posedness criteria are established.

ESAIM: Proc., Vol. 4, 1998, 269-283 


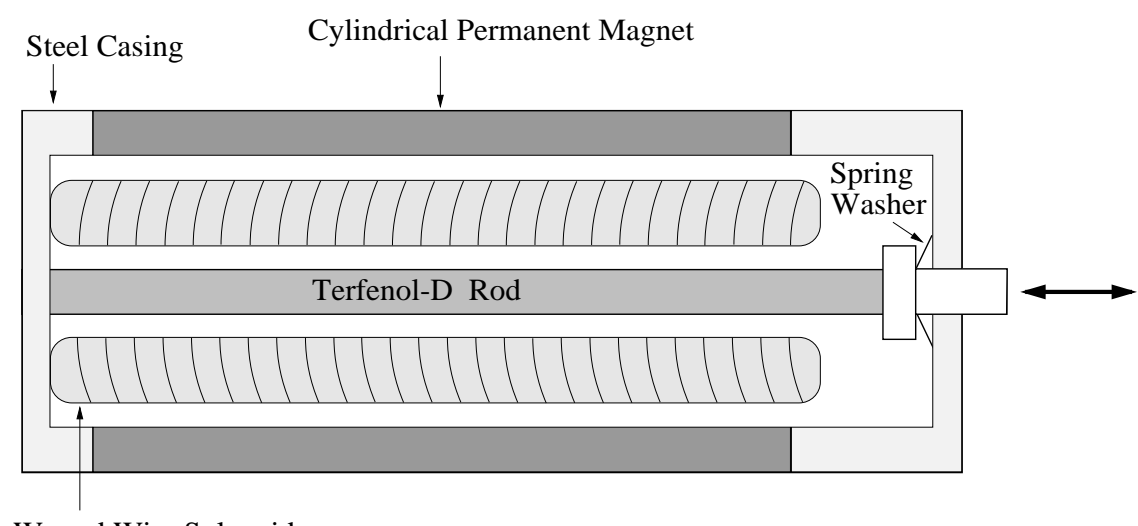

Wound Wire Solenoid

Figure 1. Cross section of a typical Terfenol-D magnetostrictive transducer.

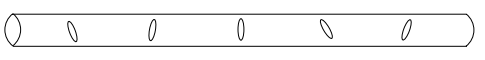

(a)

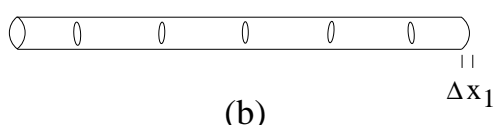

(b)

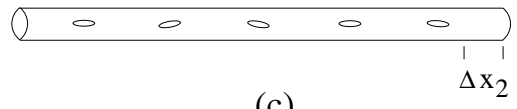

(c)

Figure 2. Magnetic moments in the Terfenol-D rod; (a) Orientation of moments in unstressed rod in absence of applied magnetic field; (b) Orientation of moments in prestressed rod with no applied field; (c) Orientation of moments in prestressed rod when field is applied in direction of longitudinal rod axis. 


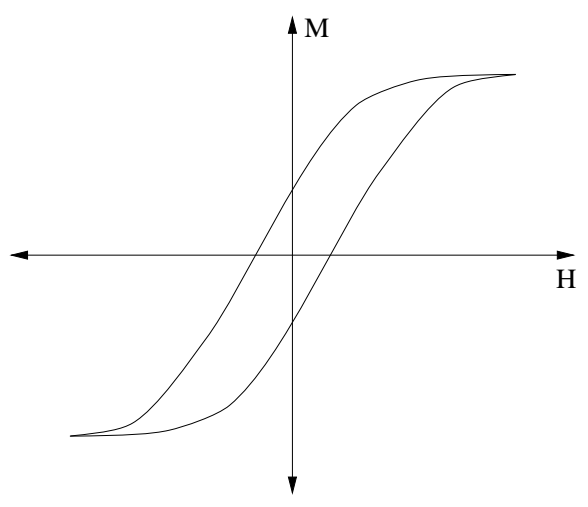

(a)

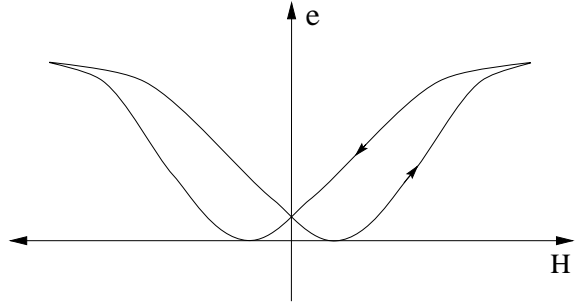

(b)

Figure 3. (a) Relationship between the magnetic field strength $H$ and the magnetization $M$; (b) Applied magnetic field $H$ and resulting strain distribution $e$.

\section{Domain wall model}

The model described here is based upon the theory that magnetization in ferromagnetic materials is due to the realignment of domains through domain wall movement in response to an applied field (domains are defined as regions in which moments are aligned). For a material which is free from defects, the domain wall movement is reversible which leads to anhysteretic (hysteresis free) behavior. Most materials, however, contain defects which impede domain wall movement and introduce hysteresis. Details regarding the physics underlying this phenomenon can be found in $[6,7,8,9]$.

To characterize the magnetization $M$, we consider first the effective field within the material. For rods subjected to a constant prestress $\sigma_{0}$, the effective field is given by

$$
H_{\text {eff }}(t)=H(t)+\alpha M(t)
$$

where

$$
H(t)=n \mathcal{I}(t)
$$

denotes the magnetic field generated by a solenoid having $n$ turns per unit length with an input current $\mathcal{I}(t)$. The parameter $\alpha$ quantifies magnetic and stress interactions. Through thermodynamic considerations, the anhysteretic magnetization is then defined in terms of the Langevin function

$$
M_{a n}(t)=M_{s}\left[\operatorname{coth}\left(\frac{H_{e f f}(t)}{a}\right)-\left(\frac{a}{H_{e f f}(t)}\right)\right] .
$$

Here $M_{s}$ denotes the saturation magnetization of the material and $a$ is a parameter which characterizes the shape of the anhysteretic curve. Energy balancing 
(see [7]) is then used to quantify the irreversible and reversible magnetizations through the expressions

$$
\frac{d M_{i r r}}{d t}=n \frac{d \mathcal{I}}{d t} \cdot \frac{M_{a n}(t)-M_{i r r}(t)}{k \delta-\alpha\left[M_{a n}(t)-M_{i r r}(t)\right]}
$$

and

$$
M_{r e v}(t)=c\left[M_{a n}(t)-M_{i r r}(t)\right]
$$

( $\delta= \pm 1$ while the constants $c$ and $k$ are estimated from the experimental hysteresis curves). Finally, the total magnetization is given by

$$
M(t)=M_{r e v}(t)+M_{i r r}(t) .
$$

To first approximation, the strains generated by the material are given by the bulk magnetostriction

$$
\lambda(t)=\frac{3}{2} \frac{\lambda_{s}}{M_{s}^{2}} M^{2}(t)
$$

where $\lambda_{s}$ denotes the saturation magnetostriction (see [6] for details). In combination, (1) - (5) characterize the relationship between the input current $\mathcal{I}$ and generated strains.

To obtain well defined magnetostrictions, and hence strains, it is necessary to specify the turning points of $\mathcal{I}$ in the manner specified in Definition 2.1 (see Figure 4). It is also necessary to restrict the admissible parameter set to guarantee that

$$
\left|k \delta-\alpha\left[M_{a n}-M_{i r r}\right]\right| \geq \varepsilon_{0}
$$

for all $t \in[0, T]$. This places a bound on the slope of the magnetization curve which is reasonable from both mathematical and physical perspectives.

Definition 2.1. Let $\mathcal{T}=\left\{t_{0}, t_{1}, t_{2}, \cdots, t_{n}\right\}$ denote the set of critical points of $\mathcal{I}$ at which $\frac{d \mathcal{I}}{d t}$ changes sign.

Proposition 2.2. Assume that (6) is satisfied, $\mathcal{I} \in C[0, T]$, and the partition $\mathcal{T}$ is specified. The differential equation (2) then has a unique continuous solution on $[0, T]$.

Proof: Equation (2) has the form

$$
\frac{\partial M_{i r r}}{\partial t}=F\left(M_{i r r}, t\right)
$$

where

$$
F\left(M_{i r r}, t\right)=n \frac{d \mathcal{I}}{d t} \cdot \frac{M_{a n}(t)-M_{i r r}(t)}{k \delta-\alpha\left[M_{a n}(t)-M_{i r r}(t)\right]} .
$$


It follows that on each subinterval $\left(t_{j}, t_{j+1}\right), j=0, \cdots, n-1, F$ and $\frac{\partial F}{\partial M_{i r r}}$ are continuous and bounded with

$$
\left|\frac{\partial F}{\partial M_{i r r}}\right| \leq \frac{k}{\varepsilon_{0}^{2}}
$$

From the theory of ordinary differential equations (e.g., see [5, Chapter 3]), it follows that (2) has a unique, continuously differentiable solution on each subinterval with well defined values at the endpoints $t_{j}$. Hence (2) has a unique solution $M_{i r r} \in C[0, T]$.

Proposition 2.3. Consider the assumptions of Proposition 2.2. It then follows that

$$
\begin{aligned}
& H \in C[0, T] \\
& M_{i r r} \in C[0, T] \\
& M_{a n} \in C[0, T] \\
& M \in C[0, T] .
\end{aligned}
$$

Proof: The result follows from the definitions of $H, M_{i r r}, M_{a n}$ and $M$ along with Proposition 2.2.

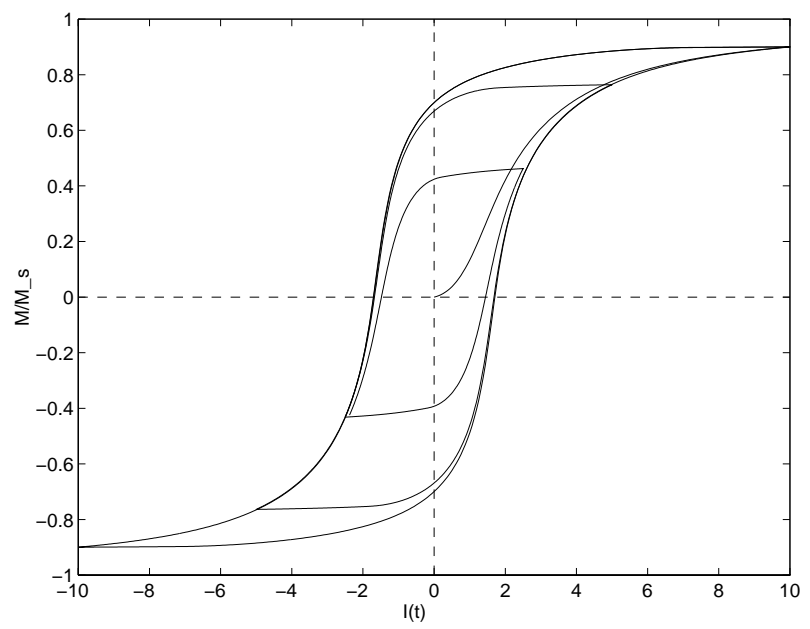

Figure 4. Nested major hysteresis loops with turning points $t_{j}$ occurring at each loop tip $\left(\frac{d \mathcal{I}}{d t}=0\right)$.

ESAIM: Proc., Vol. 4, 1998, 269-283 


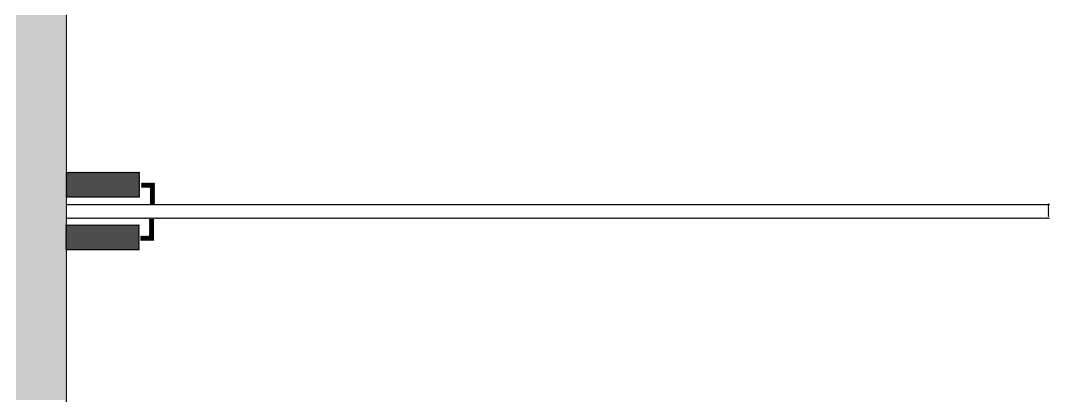

Figure 5. Cantilever beam with magnetostrictive actuators.

\section{Structural model}

To illustrate the use of magnetostrictive transducers in a structural application, we consider a cantilever beam with end-mounted actuators as depicted in Figure 5. As described in [3], this setup has been experimentally employed to ascertain properties and capabilities of the actuators.

For modeling purposes, the beam is assumed to have length $\ell$, width $b$, and thickness $h$. The density, Young's modulus, Kelvin-Voigt damping coefficient and air damping coefficient for the beam are denoted by $\rho_{b}, E_{b}, c_{D_{b}}$ and $\gamma$, respectively. The cross-sectional area of the Terfenol rod is denoted by $A_{m a g}$ while the Young's modulus and damping coefficient for the Terfenol rod are denoted by $E^{H}$ and $c_{D}^{H}$. The length and width of the connecting bar are denoted by $\ell_{r}$ and $b_{r}$, respectively, while the bar density is given by $\rho_{r}$. Finally, the transverse beam displacement is given by $w$ while $\tilde{f}(t, x)$ denotes an exogenous surface force to the beam.

We consider two models which characterize the transverse beam dynamics. The first is a modified Euler-Bernoulli model which is valid for thin structures with limited frequency ranges. Modified Timoshenko equations are also considered to provide a model valid for thick structures in which shear deformations and rotational inertia are significant. The second model should also be employed for applications which involve wide frequency responses. 


\subsection{Euler-Bernoulli model}

Moment and force balancing yields the strong form of the Euler-Bernoulli equations

$$
\begin{aligned}
& \rho(x) \frac{\partial^{2} w}{\partial t^{2}}(t, x)+\gamma \frac{\partial w}{\partial t}(t, x)+\frac{\partial^{2} \mathcal{M}_{i n t}}{\partial x^{2}}(t, x)=\tilde{f}(t, x)+\frac{\partial^{2} \mathcal{M}_{\text {mag }}}{\partial x^{2}}(t, x) \\
& , \text { with } \begin{array}{l}
0<x<\ell \\
t>0
\end{array} \\
& \left.\begin{array}{l}
w(t, 0)=\frac{\partial w}{\partial x}(t, 0)=0 \\
\mathcal{M}_{\text {int }}(t, \ell)=\frac{\partial \mathcal{M}_{\text {int }}}{\partial x}(t, \ell)=0
\end{array}\right\}, t>0,
\end{aligned}
$$

along with appropriate initial conditions, as a model for characterizing the transverse beam dynamics. As detailed in [9], the composite density and internal bending moment are given by

$$
\begin{aligned}
& \rho(x)=\rho_{b} h b+2 \rho_{r} b_{r} \ell_{r} \chi_{\text {rod }}(x) \\
& \mathcal{M}_{\text {int }}(t, x)=E I(x) \frac{\partial^{2} w}{\partial x^{2}}(t, x)+c_{D} I \frac{\partial^{3} w}{\partial x^{2} \partial t}(t, x) .
\end{aligned}
$$

where the characteristic function $\chi_{\text {rod }}$ delineates the location of the rods and

$$
\begin{aligned}
& E I(x)=\frac{E_{b} h^{3} b}{12}+2 A_{\text {mag }} E^{H}\left(h / 2+\ell_{r}\right)^{2} \chi_{\text {rod }}(x) \\
& c_{D} I(x)=\frac{c_{D_{b}} h^{3} b}{12}+2 A_{\text {mag }} c_{D}^{H}\left(h / 2+\ell_{r}\right)^{2} \chi_{\text {rod }}(x) .
\end{aligned}
$$

For the case when the Terfenol rods are driven diametrically out-of-phase, the external moment is given by

$$
\mathcal{M}_{\text {mag }}(t, x)=2 A_{\text {mag }} E^{H}\left(h / 2+\ell_{r}\right)^{2} \lambda(t) \chi_{\text {rod }}(x)
$$

where the magnetostriction $\lambda$ is given by (5).

In order to obtain a weak form of the model, we take the state to be the displacement $w$ in the state space $X=L^{2}(0, \ell)$ with the inner product

$$
\langle\phi, \psi\rangle_{X}=\int_{0}^{\ell} \rho \phi \psi d x .
$$

The space of test functions is taken to be $V=H_{L}^{2}(0, \ell) \equiv\left\{\phi \in H^{2}(0, \ell) \mid \phi(0)=\right.$ $\left.\phi^{\prime}(0)=0\right\}$ with the inner product

$$
\langle\phi, \psi\rangle_{V}=\int_{0}^{\ell} E I \phi^{\prime \prime} \psi^{\prime \prime} d x .
$$

ESAIM: Proc., Vol. 4, 1998, 269-283 
It should be noted that with these choices, $V$ is continuously and densely embedded in $H$. Hence one has the Gelfand triple

$$
V \hookrightarrow X \simeq X^{*} \hookrightarrow V^{*}
$$

with the pivot space $X$. A weak form of the model is then given by

$$
\int_{0}^{\ell} \rho \ddot{w} \psi d x+\int_{0}^{\ell} \gamma \dot{w} \psi d x+\int_{0}^{\ell} \mathcal{M}_{i n t} \psi^{\prime \prime} d x=\int_{0}^{\ell} \mathcal{M}_{m a g} \psi^{\prime \prime} d x+\int_{0}^{\ell} \tilde{f} \psi d x
$$

for all $\psi \in V$.

To provide an abstract framework amenable to well-posedness analysis, we define the sesquilinear forms

$$
\begin{aligned}
& \sigma_{1}(\phi, \psi)=\int_{0}^{\ell} E I \phi^{\prime \prime} \psi^{\prime \prime} d x \\
& \sigma_{1}(\phi, \psi)=\int_{0}^{\ell} c_{D} I \phi^{\prime \prime} \psi^{\prime \prime} d x+\int_{0}^{\ell} \gamma \phi \psi d x .
\end{aligned}
$$

It can be directly verified that $\sigma_{1}$ and $\sigma_{2}$ satisfy the boundedness and ellipticity conditions

$$
\begin{aligned}
& \left|\sigma_{1}(\phi, \psi)\right| \leq c_{1}|\phi|_{V}|\psi|_{V}, c_{1} \in \mathbb{R} \\
& \operatorname{Re} \sigma_{1}(\phi, \phi) \geq c_{2}|\phi|_{V}^{2}, c_{2}>0 \\
& \left|\sigma_{2}(\phi, \psi)\right| \leq c_{3}|\phi|_{V}|\psi|_{V}, c_{3} \in \mathbb{R} \\
& \operatorname{Re} \sigma_{2}(\phi, \phi)+c_{4}|\phi|_{H}^{2} \geq c_{5}|\phi|_{V}^{2}, c_{4} \in \mathbb{R}, c_{5}>0
\end{aligned}
$$

for all $\phi, \psi \in V$. The magnetostrictive actuator inputs are represented by the operator $B: U \rightarrow V^{*}$ given by

$$
\begin{aligned}
\langle[B(\mathcal{I})](t), \psi\rangle_{V^{*}, V} & \equiv \int_{0}^{\ell} \mathcal{M}_{\text {mag }}(t, x) \psi^{\prime \prime} d x \\
& =\int_{\text {rod }} 3 A_{\text {mag }} E^{H}\left(h / 2+\ell_{r}\right)^{2} \frac{\lambda_{s}}{M_{s}^{2}} M^{2}(t) \psi^{\prime \prime} d x
\end{aligned}
$$

where $U \equiv \mathbb{R}$ and $\langle\cdot, \cdot\rangle_{V^{*}, V}$ denotes the usual duality product. Finally, with the definition $f(t)=\left(\rho^{-1} \tilde{f}(t, \cdot), 0\right)$, we can write the weak form (11) in the abstract variational form

$$
\begin{aligned}
& \langle\ddot{w}(t), \psi\rangle_{V^{*}, V}+\sigma_{2}(\dot{w}(t), \psi)+\sigma_{1}(w(t), \psi)=\langle[B(\mathcal{I})](t)+f(t), \psi\rangle_{V^{*}, V}, \forall \psi \in V \\
& w(0)=w_{0} \quad, \quad \dot{w}(0)=w_{1} .
\end{aligned}
$$


An equivalent system can be obtained by invoking the boundedness of $\sigma_{1}, \sigma_{2}$ to define operators $A_{i} \in \mathcal{L}\left(V, V^{*}\right), i=1,2$, by

$$
\begin{aligned}
& \left\langle A_{1} \phi, \psi\right\rangle_{V^{*}, V}=\sigma_{1}(\phi, \psi) \\
& \left\langle A_{2} \phi, \psi\right\rangle_{V^{*}, V}=\sigma_{2}(\phi, \psi)
\end{aligned} \quad, \quad \phi, \psi \in V .
$$

In operator form, the equations governing the beam dynamics then have the form

$$
\ddot{w}(t)+A_{2} \dot{w}(t)+A_{1} w(t)=[B(\mathcal{I})](t)+f(t)
$$

in $V^{*}$. Subsequent well-posedness results are established in the following theorem.

Lemma 3.1. Define the operator $B$ by (13) and let $\mathcal{T}$ denote the partition described by Definition 2.1. Under the assumption that $\mathcal{I} \in C[0, T]$ and that the parameters satisfy (6), it follows that

$$
B(\mathcal{I}) \in L^{2}\left((0, T) ; V^{*}\right) .
$$

Proof: The operator $B$ in (13) can be decomposed as

$$
[B(\mathcal{I})](t)=[P(\mathcal{I})](t) \cdot g \quad, \quad g \in V^{*}
$$

where

$$
\begin{aligned}
& {[P(\mathcal{I})](t)=3 A_{\text {mag }} E^{H}\left(h / 2+\ell_{r}\right)^{2} \frac{\lambda_{s}}{M_{s}^{2}} M^{2}(t)} \\
& g(v)=\int_{\text {rod }} v^{\prime \prime} d x .
\end{aligned}
$$

From Proposition 2.3, it follows that

$$
P(\cdot): C[0, T] \rightarrow C[0, T]
$$

so that the norm

$$
\|[B(\mathcal{I})](t)\|_{V^{*}}=\sup _{v \in V} \frac{|[P(\mathcal{I})](t) \cdot g(v)|}{\|v\|_{V}}
$$

exists for each $t \in[0, T]$. Since $\|[B(\mathcal{I})](t)\|_{V^{*}}=|[P(\mathcal{I})](t)| \cdot\|g\|_{V^{*}}$, it follows that

$$
\|B(\mathcal{I})\|_{L^{2}\left((0, T) ; V^{*}\right)}^{2} \leq \max _{t \in[0, T]}\left\{|[P(\mathcal{I})](t)|^{2}\right\} \cdot T \cdot\|g\|_{V^{*}}^{2}
$$

which implies that

$$
B(\mathcal{I}) \in L^{2}\left((0, T) ; V^{*}\right)
$$

ESAIM: Proc., Vol. 4, 1998, 269-283 
Theorem 3.2. Let $\sigma_{1}, \sigma_{2}$ be given by (12) and consider the assumptions of Lemma 3.1. There then exists a unique solution $w$ to (14) which satisfies

$$
\begin{aligned}
& w \in C((0, T) ; V) \\
& \dot{w} \in C((0, T) ; H) .
\end{aligned}
$$

Proof: The result follows directly from Theorem 4.1 of [2] or Theorem 2.1 and Remark 2.1 of [1].

\subsection{Timoshenko model}

A more general characterization of the structural dynamics, which includes the effects of shear deformation and rotational inertia, is provided by a Timoshenko model. For this model, we take the states to be $w$ and $\theta$ where $w$ again denotes the transverse displacement and $\theta$ is the cross sectional rotation. As detailed in [10], an appropriate strong form of the structural model is then

$$
\begin{aligned}
& \left.\begin{array}{l}
\rho(x) \frac{\partial^{2} w}{\partial t^{2}}(t, x)+\gamma \frac{\partial w}{\partial t}(t, x)-\frac{\partial \mathcal{Q}}{\partial x}(t, x)=\tilde{f}(t, x) \\
\rho r(x) \frac{\partial^{2} \theta}{\partial t^{2}}(t, x)-\frac{\partial \mathcal{M}_{\text {int }}}{\partial x}(t, x)+\mathcal{Q}(t, x)=\frac{-\partial \mathcal{M}_{\text {mag }}}{\partial x}(t, x)
\end{array}\right\} \quad \begin{array}{c}
0<x<\ell \\
t>0
\end{array} \\
& \left.\begin{array}{l}
w(t, 0)=\theta(t, 0)=0 \\
\mathcal{M}(t, \ell)=\mathcal{Q}(t, \ell)=0
\end{array}\right\}, t>0
\end{aligned}
$$

along with appropriate initial conditions. The composite density $\rho(x)$ is defined in (7) while $r(x) \equiv I(x) / A(x)$ where $A(x)$ and $I(x)$ respectively denote the cross sectional area and moment of inertia. The internal moment and shear are given by

$$
\begin{aligned}
& \mathcal{M}_{\text {int }}(t, x)=E I(x) \frac{\partial \theta}{\partial x}(t, x)+c_{D} I(x) \frac{\partial^{2} \theta}{\partial x \partial t}(t, x) \\
& \mathcal{Q}(t, x)=\kappa A G(x) \beta(t, x)+\kappa A c_{Q}(x) \frac{\partial \beta}{\partial t}(t, x)
\end{aligned}
$$

where $\kappa$ is a correction factor which accounts for the fact that the outer surface of the beam cannot support a shear stress, $G(x)$ is the shear modulus and $c_{Q}(x)$ represents resistance to the shear strain rate. The stiffness and damping parameters $E I(x)$ and $c_{D} I(x)$ are defined in (8). The shear deformation is given by

$$
\beta(t, x)=\frac{\partial w}{\partial x}(t, x)+\theta(t, x)
$$

while the external moment $\mathcal{M}_{\text {mag }}$ is defined in (9). 
To obtain an appropriate weak form of the model, the state $y=(w, \theta)$ is considered in the Hilbert space $X=L^{2}(0, \ell) \times L^{2}(0, \ell)$ with the inner product

$$
\langle\phi, \psi\rangle_{X}=\int_{0}^{\ell} \rho \phi_{1} \psi_{1} d x+\int_{0}^{\ell} \rho r \phi_{2} \psi_{2} d x
$$

where $\phi=\left(\phi_{1}, \phi_{2}\right), \psi=\left(\psi_{1}, \psi_{2}\right)$. The space of test functions is $V=H_{L}^{1}(0, \ell) \times$ $H_{L}^{1}(0, \ell), H_{L}^{1}(0, \ell) \equiv\left\{\eta \in H^{1}(0, \ell) \mid \eta(0)=0\right\}$, with the inner product

$$
\langle\phi, \psi\rangle_{V}=\int_{0}^{\ell} \kappa A G\left(\phi_{1}^{\prime}+\phi_{2}\right)\left(\psi_{1}^{\prime}+\psi_{2}\right) d x+\int_{0}^{\ell} E I \phi_{2}^{\prime} \psi_{2}^{\prime} d x .
$$

It should be noted that with these choices, $V$ and $H$ again form a Gelfand triple (10). A weak form of the modeling equations is then

$$
\begin{gathered}
\int_{0}^{\ell} \rho \ddot{w} \psi_{1} d x+\int_{0}^{\ell} \gamma \dot{w} \psi_{1} d x+\int_{0}^{\ell} \mathcal{Q} \psi_{1}^{\prime} d x+\int_{0}^{\ell} \rho r \ddot{\theta} \psi_{2} d x+\int_{0}^{\ell} \mathcal{M}_{i n t} \psi_{2}^{\prime} d x \\
+\int_{0}^{\ell} \mathcal{Q} \psi_{2} d x=\int_{0}^{\ell} \mathcal{M}_{m a g} \psi_{2}^{\prime} d x+\int_{0}^{\ell} \tilde{f} \psi_{1} d x
\end{gathered}
$$

for all $\psi \in V$.

Sesquilinear forms for this case are given by

$$
\begin{aligned}
& \sigma_{1}(\phi, \psi)=\int_{0}^{\ell} E I \phi_{2}^{\prime} \psi_{2}^{\prime} d x+\int_{0}^{\ell} \kappa A G\left(\phi_{1}^{\prime}+\phi_{2}\right)\left(\psi_{1}^{\prime}+\psi_{2}\right) d x \\
& \sigma_{2}(\phi, \psi)=\int_{0}^{\ell} c_{D} I \phi_{2}^{\prime} \psi_{2}^{\prime} d x+\int_{0}^{\ell} \kappa A c_{Q}\left(\phi_{1}^{\prime}+\phi_{2}\right)\left(\psi_{1}^{\prime}+\psi_{2}\right) d x+\int_{0}^{\ell} \gamma \phi_{1} \psi_{1} d x
\end{aligned}
$$

where again, $\phi=\left(\phi_{1}, \phi_{2}\right), \psi=\left(\psi_{1}, \psi_{2}\right)$. It can be directly verified that $\sigma_{1}$ and $\sigma_{2}$ satisfy the conditions (H1) - (H4) and hence generate operators $A_{1}, A_{2} \in \mathcal{L}\left(V, V^{*}\right)$. By defining inputs through the operator definition (13), the Timoshenko model can be written in the abstract form (14) or (15). The well-posedness of the model is then obtained in the manner specified in Theorem 3.2 for the Euler-Bernoulli model.

\section{Concluding remarks}

This paper addresses issues which arise when modeling magnetostrictive actuator inputs to structural systems. These actuators utilize the property that strains in the material are generated in response to an applied magnetic field. The advantage of these materials lies in the large magnitude of the generated strains and forces. In the regime where such forces and strains are generated, however, the materials exhibit significant nonlinearities and hysteresis. The material dynamics are characterized here through an energy model based upon

ESAIM: Proc., Vol. 4, 1998, 269-283 
domain wall properties. This provides an actuator model which is accurate for a variety of operating conditions. The use of this model is illustrated in the context of Euler-Bernoulli and Timoshenko beam models. It is demonstrated that the models are well-posed when input currents are continuous. This provides a characterization of the magnetostrictive actuators which is suitable for ensuing control applications.

\section{Acknowledgements}

The author would like to thank F.T. Calkins and D.C. Jiles for input regarding the modeling techniques employed here. Sincere appreciation is also extended to the organizing committee for the planning and assistance provided during this conference. This research was supported in part by NASA grant NAG-1-1600.

\section{References}

[1] H.T. Banks, K. Ito and Y. Wang, "Well-Posedness for Damped Second Order Systems with Unbounded Input Operators," Differential and Integral Equations, 8, pp. 587-606, 1995.

[2] H.T. Banks, R.C. Smith and Y. Wang, Smart Material Structures: Modeling, Estimation and Control, Masson/John Wiley, Paris/Chichester, 1996.

[3] F.T. Calkins, R.L. Zrostlik and A.B. Flatau, "Terfenol-D Vibration Control of a Rotating Shaft," Proceedings of the 1994 ASME International Mechanical Engineering Congress and Exposition, Chicago IL; In Adaptive Structures and Composite Materials Analysis and Applications AD-Vol. 45, pp. 267-274, 1996.

[4] D.L. Hall and A.B. Flatau, "Nonlinearities, Harmonics and Trends in Dynamic Applications of Terfenol-D," Proceedings of the SPIE Conference on Smart Structures and Intelligent Materials, Vol. 1917, Part 2, pp. 929-939, 1993.

[5] E.L. Ince, Ordinary Differential Equations, Dover Publications, Inc., New York, 1956.

[6] D.C. Jiles, Introduction to Magnetism and Magnetic Materials, Chapman and Hall, New York, 1991.

[7] D.C. Jiles and D.L. Atherton, "Theory of Ferromagnetic Hysteresis," Journal of Magnetism and Magnetic Materials, 61, pp. 48-60, 1986.

[8] D.C. Jiles, J.B. Thoelke and M.K. Devine, "Numerical Determination of Hysteresis Parameters for the Modeling of Magnetic Properties Using the Theory of Ferromagnetic Hysteresis," IEEE Transactions on Magnetics, 28(1), pp. 27-35, 1992. 
[9] R.C. Smith, "Modeling Techniques for Magnetostrictive Actuators," CRSC Technical Report CRSC-TR97-6; Proceedings of the SPIE, Smart Structures and Integrated Systems, San Diego, CA, March 1997, Vol. 3041, pp. 243-253.

[10] R.C. Smith, "Hysteresis Modeling in Magnetostrictive Materials Via Preisach Operators," ICASE Report 97-23; Journal of Mathematical Systems, Estimation and Control, to appear. 